\title{
Análisis crítico de un artículo
}

\section{Las estatinas reducen la morbi-mortalidad en pacientes de alto riesgo cardiovascular}

\author{
Tomás Pantoja1 \\ Luz María Letelier²,3
}

\author{
MRC/BHF Heart Protection Study of cholesterol lowering \\ with simvastatin in 20,536 high-risk individuals: a \\ randomized placebo-controlled trial. \\ Heart Protection Study Collaborative Group. (HPS). \\ Lancet 2002; 360: 7-22.
}

Background: Throughout the usual LDL cholesterol range in Western populations, lower blood concentrations are associated with lower cardiovascular disease risk. In such populations, therefore, reducing LDL cholesterol may reduce the development of vascular disease, largely irrespective of initial cholesterol concentrations. Methods: 20,536 UK adults (aged 40-80 years) with coronary disease, other occlusive arterial disease, or diabetes were randomly allocated to receive $40 \mathrm{mg}$ simvastatin daily (average compliance: 85\%) or matching placebo (average non-study statin use: 17\%). Analyses are of the first occurrence of particular events, and compare all simvastatin-allocated versus all placebo-allocated participants. These «ntention-to-treat»comparisons assess the effects of about two-thirds (85\% minus 17\%) taking a statin during the scheduled 5-year treatment period, which yielded an average difference in LDL cholesterol of $1.0 \mathrm{mmol} / \mathrm{L}$ (about two-thirds of the effect of actual use of $40 \mathrm{mg}$ simvastatin daily). Primary outcomes were mortality (for overall analyses) and fatal or non-fatal vascular events (for subcategory analyses), with subsidiary assessments of cancer and of other major morbidity. Findings: All-cause mortality was significantly reduced $(1,328$ [12.9\%] deaths among 10,269 allocated simvastatin versus 1,507 [14.7\%] among 10,267 allocated placebo; $\mathrm{p}=0.0003$ ), due to a highly significant $18 \%$ (SE 5) proportional reduction in the coronary death rate (587 [5.7\%] vs 707 [6.9\%]; $\mathrm{p}=0.0005)$, a marginally significant reduction in other vascular deaths (194 [1.9\%] vs 230 [2.2\%]; $\mathrm{p}=0.07)$, and a non-significant reduction in non-vascular deaths (547 [5.3\%] vs 570 [5.6\%]; $p=0.4$ ). There were highly significant reductions of about onequarter in the first event rate for non-fatal myocardial infarction or coronary death (898 [8.7\%] vs 1212 [11.8\%]; $\mathrm{p}<0.0001$ ), for non-fatal or fatal stroke (444 [4.3\%] vs 585 [5.7\%] $\mathrm{p}<0.0001$ ), and for coronary or non-coronary revascularisation (939 [9.1\%] vs 1205 [11.7\%]; $\mathrm{p}<0.0001)$. For the first occurrence of any of these major vascular events, there was a definite $24 \%$ (SE 3; 95\% CI 19-28) reduction in the event rate (2033 [19.8\%] vs 2585 [25.2\%] affected individuals; $\mathrm{p}<0.0001$ ). During the first year the reduction in major vascular events was not significant, but subsequently it was highly significant during each separate year. The proportional reduction in the event rate was similar (and significant) in each subcategory of participant studied, includ- 
ing: those without diagnosed coronary disease who had cerebrovascular disease, or had peripheral artery disease, or had diabetes; men and, separately, women; those aged either under or over 70 years at entry; and-most notably-even those who presented with LDL cholesterol below $3.0 \mathrm{mmol} / \mathrm{L}$ (116 mg/dL), or total cholesterol below $5.0 \mathrm{mmol} / \mathrm{L}(193 \mathrm{mg} / \mathrm{dL})$. The benefits of simvastatin were additional to those of other cardioprotective treatments. The annual excess risk of myopathy with this regimen was about $0.01 \%$. There were no significant adverse effects on cancer incidence or on hospitalisation for any other non-vascular cause. Interpretation: Adding simvastatin to existing treatments safely produces substantial additional benefits for a wide range of high-risk patients, irrespective of their initial cholesterol concentrations. Allocation to $40 \mathrm{mg}$ simvastatin daily reduced the rates of myocardial infarction, of stroke, and of revascularisation by about one-quarter. After making allowance for non-compliance, actual use of this regimen would probably reduce these rates by about one-third. Hence, among the many types of high-risk individual studied, 5 years of simvastatin would prevent about 70-100 people per 1000 from suffering at least one of these major vascular events (and longer treatment should produce further benefit). The size of the 5-year ben efit depends chiefly on such individuals' overall risk of major vascular events, rather than on their blood lipid concentrations alone.

${ }^{1}$ Departamento de Medicina Familiar, Pontificia Universidad Católica de Chile.

2 Departamento de Medicina Interna, Pontificia Universidad Católica de Chile.

${ }^{3}$ Servicio de Medicina, Hospital Sótero del Río. Santiago de Chile.

Revisado por Unidad de Medicina Basada en Evidencia UC (UMBE-UC).

\section{CONCLUSIÓN DE LOS REVISORES:}

La simvastatina sumada al tratamiento estándar, reduce la mortalidad global y la morbimortalidad cardiovascular en una gran variedad de pacientes de alto riesgo cardiovascular, independientemente del nivel de colesterol.

\section{PREgUNTA CLÍNICA:}

¿Se reducen la mortalidad global y la morbimortalidad cardiovascular en pacientes de alto riesgo cardiovascular si indico simvastatina?

\section{ConteXto:}

Está bien establecida la relación entre el aumento de los niveles de colesterol, en particular del colesterol LDL y el aumento de riesgo cardiovascular. Las estatinas han sido recomendadas en la prevención primaria ${ }^{1}$ y secundaria ${ }^{2}$ de eventos coronarios en poblaciones con niveles de colesterol elevado. También se han utilizado en pacientes con niveles normales de colesterol y enfermedad coronaria demostrada ${ }^{3}$. Sin embargo, la evidencia es aún limitada en ciertos grupos de alto riesgo como mujeres, adultos mayores, y en aquellos con niveles de colesterol en rangos normales sin enfermedad coronaria demostrada. 


\section{CARACTERÍSTICAS DEL ESTUDIO:}

Pacientes: 20.536 hombres y mujeres entre 40 y 80 años con nivel de colesterol total mayor a $135 \mathrm{mg} / \mathrm{dL}$ y con enfermedad coronaria o enfermedad arterial oclusiva no coronaria, o diabetes o sólo hipertensión si eran hombres mayores de 65 años. Se excluyeron pacientes con patología renal, hepática o muscular. Intervención: 10.269 pacientes asignados a $40 \mathrm{mg} /$ día de simvastatina vía oral. Comparación: 10.267 pacientes asignados a placebo. Outcomes: Primarios: Mortalidad general, mortalidad por enfermedad coronaria y mortalidad por causas no coronarias. Secundarios: Eventos coronarios mayores y eventos vasculares mayores. Seguimiento: promedio 5 años.

\section{VALDEZ:}

1. Randomizado:

2. Secuencia de asignación oculta:

3. Porcentaje de seguimiento:

4. Análisis con intención de tratar:

5. Grupos similares respecto a variables pronósticas conocidas

SI

6. Fueron ciegos a la intervención:

Pacientes:

Tratantes:

Recolectores de datos:

Adjudicadores de eventos:

Analistas de datos:

cierto

REsultados

\begin{tabular}{|lcccc|}
\hline Resultados & $\begin{array}{c}\text { Tasa de eventos } \\
\text { grupo simvastatina }\end{array}$ & $\begin{array}{c}\text { Tasa de eventos } \\
\text { grupo placebo }\end{array}$ & $\begin{array}{c}\text { RRR } \\
\text { (IC 95\%) }\end{array}$ & $\begin{array}{c}\text { NNT } \\
\text { (IC 95\%) }\end{array}$ \\
\hline Mortalidad general & $12,9 \%$ & $14,7 \%$ & $\begin{array}{c}13 \% \\
(6 \% \text { a } 19 \%)\end{array}$ & $\begin{array}{c}56 \\
(37-117)\end{array}$ \\
$\begin{array}{l}\text { Mortalidad por eventos } \\
\text { coronarios }\end{array}$ & $5,7 \%$ & $6,9 \%$ & $\begin{array}{c}18 \% \\
(9 \% \text { a } 27 \%)\end{array}$ & $\begin{array}{c}(54-189) \\
\text { Mortalidad no vascular }\end{array}$ \\
$\begin{array}{l}\text { Primer evento vascular } \\
\text { mayor** }\end{array}$ & $19,8 \%$ & $5,6 \%$ & $5 \%$ & 333 \\
\end{tabular}

$\mathrm{RRR}=$ Reducción relativa de riesgo. $\mathrm{NNT}=$ Número necesario para tratar.

IC= Intervalo de confianza.

$*$ infinito

** incluye eventos coronarios mayores, cualquier evento cerebro vascular y revascularización coronaria o no coronaria. 


\section{COMENTARIOS Y APLICACIÓN PRÁCTICA:}

- El estudio no tiene amenazas evidentes a su validez interna e incluyó una gran variedad de pacientes de alto riesgo cardiovascular, incluyendo $25 \%$ de mujeres.

- Hubo una clara reducción de la morbimortalidad cardiovascular y de la mortalidad general al agregar simvastatina al tratamiento habitual de aspirina, betabloqueadores, e inhibidores de enzima convertidora (IECA), independiente de los niveles iniciales de colesterol. El análisis de los resultados muestra que bastaría tratar 56 y 19 pacientes similares a los del estudio por 5 años con simvastatina para evitar una muerte y un primer evento vascular mayor, respectivamente. No se demuestra en cambio efecto sobre la mortalidad no cardiovascular. Se debe destacar que el beneficio se aprecia sólo después del primer año de uso de estatinas.

- A la fecha de inicio del estudio sólo 63\% tomaba aspirina, 26\% betabloqueadores y 19\% IECA, por lo cual no sabemos cuántos estaban recibiendo la terapia actualmente considerada estándar, esto podría modificar la magnitud del beneficio. Por otra parte, al término del estudio $82 \%$ de los pacientes asignados a simvastatina seguían tomándola y $32 \%$ de los asignados a placebo estaban recibiendo alguna estatina, el análisis por intención de tratar utilizado en este estudio puede subestimar el efecto potencial.

- El estudio no demostró aumento de efectos adversos, pero el período de «un in» estipulado en el diseño permitió excluir inicialmente a los pacientes con reacciones adversas al medicamento, por lo que el estudio no permite sacar conclusiones respecto de la real incidencia de efectos adversos.

- Aunque este estudio se realizó con simvastatina, estudios previos en otras poblaciones $^{1-3}$ han demostrado beneficios con otras estatinas, pudiendo suponerse un efecto de clase de estas drogas.

\section{REFERENCIAS}

1. Pignone M, Phimps C, Mulrow C. Use of lipid lowering drugs for primary prevention of coronary heart disease: meta-analysis of randomised trials. BMJ 2000; 321: 1-5.

2. Shepherd J, Cobbe SM, Ford I ET al. For the West of Scotland Coronary Prevention Study Group. Prevention of coronary heart disease with pravastatin in men with hypercholesterolemia. N Engl J Med 1995; 333: 1301-7.

3. The Long Term Intervention With Pravastatin In Ischaemic Disease (LIPID) Study Group. Prevention of cardiovascular events and death with pravastatin in patients with coronary heart disease and a broad range of initial cholesterol levels. N Engl J Med 1998; 339: 1349-57. 